\title{
Application of New Biomedical Materials in Orthodontic Appliances
}

\author{
Hafiz Taha Mahmood1, Adeel Tahir Kamal1, Bushra Nazeer Khan² and Mubassar Fida1
}

\begin{abstract}
This review article describes the application and characteristics of certain biomedical materials in orthodontic appliances. The elastic recoil of shape memory polymers, determination of the forces and moments experienced by the brackets and eventually by the tooth, reduction in treatment time by employing self-healing smart brackets and decreased enamel lost during debonding due to usage of biomimetic adhesives such as dihydroxyphenylalanine (DOPA) is discussed. Increased plaque retention and microbial attachment around brackets and teeth is of profound concern and by utilisation of hydrophobic properties of self-cleaning materials, this can be reduced significantly. Implantation of bioresorbable temporary anchorage devices, which resorb once their purpose is accomplished and increasing the concentration of fluoride in the oral environment to counter the deleterious consequences of orthodontic treatment such as white spot lesions and caries, are also discussed briefly.
\end{abstract}

Key Words: Biomedical engineering, Orthodontic appliances, Biodegradable materials.

\section{INTRODUCTION}

The importance of a well-coordinated interaction of orthodontists with biomedical engineers for the production of many customised orthodontic appliances has been underappreciated. Usually, the biomedical engineers, hired by many orthodontic appliance manufacturing companies, develop the appliances, overlooking the day-to-day clinical difficulties faced by the orthodontists in their busy practices. On the other hand, not many general dentists choose or pursue their career in biomedical engineering as well. In addition, an absence of collaboration of orthodontists with biomedical engineers in their research endeavours leads to a significant vacuum in the innovation of biomedical materials in the orthodontic science. 1

Numerous appliances and materials are introduced in the market and yet they fail to influence orthodontic practices. The innovation should address a critical clinical concern; for example, a material that has easy handling characteristics and shortens chair side time or one that significantly improves the quality of treatment by addressing important variables such as treatment duration and cost. ${ }^{2}$ Moreover, a material which can help reduce errors in treatment will be widely accepted by the orthodontic community. Clinician's experience has

1 Department of Surgery, Aga Khan University Hospital, Karachi, Pakistan

2 Department of Orthodontics, Altamash Institute of Dental Medicine, Karachi, Pakistan

Correspondence: Dr. Mubassar Fida, Department of Surgery, Section of Dentistry, Aga Khan University Hospital, Karachi, Pakistan

E-mail: mubassar.fida@aku.edu

Received: September 05, 2018; Revised: December 18, 2018; Accepted: December 19, 2018 played a vital role in the development of many materials that are being used in orthodontic practices today. ${ }^{3}$

Technological advances have helped develop complete orthodontic solutions such as Suresmile, Invisalign and Incognito systems. ${ }^{4,5}$ Each of these offer crucial benefits to the patient and to the clinician and have been refined through close communication and interaction with the orthodontists to reach exemplary finesse.

The aim of this review is to describe the current trends and innovations of biomedical materials and their implications in orthodontic science. The list of new biomedical materials is fairly extensive and we cannot discuss all the innovations related to the orthodontic science. Hence, only the materials that could overcome day-to-day clinical problems are discussed.

Shape-memory polymers (SMPs): Polymers continue to be an important component of a variety of materials used in orthodontics, let alone dentistry. Their wide clinical application has proved to be most useful in several circumstances.

The metallic color of orthodontic appliances such as brackets and archwires has always been a great concern in adolescents and adult orthodontic patients. Means to make appliances more esthetic have led to the employment of ceramic, plastic or polycarbonate brackets and Teflon coated archwires for esthetically concerned patients. ${ }^{6}$

SMPs, as the name suggests, are the polymers that attain their original shape when deformed due to the application of a recovery trigger in the form of either light, heat, electrical or magnetic fields, infrared radiation, or immersion in water.7 Due to interatomic robust and directional bonds, SMPs can remember its original shape and return back to it, in turn having a dual shape capability. 
SMPs have numerous inherent advantages, such as transparent nature, easy working, low density, reduced cost, and acceptable appearance. 8 In addition, SMPs have a considerable shape returning force for approximately 3 months. 9 They were introduced in orthodontic treatment for initial alignment and levelling of esthetically concerned patients. 10 SMPs consists of hard and soft segments and have gained increased popularity among many clinicians due to their glass transition temperature, which is near the body temperature.11 These polymers have increased utility in the correction of malaligned and severely rotated teeth.

Brackets with force moment sensors: When a twocouple system or an indeterminate force system is employed using orthodontic appliances, the amount of forces and moments cannot be measured or appropriately determined. This drawback and with the frequent utilisation of two-couple system in orthodontics, researchers are constantly looking for modalities or devices that can measure these forces and moments first in vitro and later on, in vivo. Hence, there will be adequate utilisation of forces for effective orthodontic tooth movement and minimal consequences on oral health. 12 However, in vitro assessment has certain limitations, because, by all means, we cannot provide the exact environment imitating the oral apparatus.

According to the pertinent literature survey, many in vitro, ${ }^{13,14}$ and later in vivo ${ }^{15}$ systems have been introduced in the past to precisely measure forces and moments. In the last decade, Lapatki et al. introduced a smart bracket having stress sensor system implanted in the bracket base for the evaluation of three dimensional forces and moments experienced by the bracket and then eventually by the tooth. ${ }^{15}$ However, none of the systems have yet been tested and used in the oral environment; and further research in this regard is essential.

Self-healing materials: The development of smart synthetic materials, which show prompt reactions to environmental behaviours, have been an interesting innovation because of their unusual biomimetic properties. ${ }^{12}$ Motivated by nature, researchers have developed some smart materials which have selfhealing properties, capable of healing the damage and applicable during drug delivery, tissue and organ repairing and shape memory functions. ${ }^{16}$ Over the past few decades, hydrogels have been introduced, which display unusual bio-mimicking properties. Researchers demonstrated some cross-linked hydrogels which show self-healing properties. ${ }^{17}$

Self-healing materials for orthodontic applications can include polymer brackets and archwires. Wire and bracket breakages can be minimised by incorporating nanosized bubbles filled with auto-polymerised monomer. When a bracket breaks, shattering of the bubbles take place which leads the monomer out into the air; as a consequence of which, space created by fracture is polymerised and filled. 12 This would result in minimising the damage of the brackets and archwires and simultaneously leads to an overall reduction in treatment duration. However, multiple trials and approval from different organisations such as Current Good Manufacturing Practice (CGMP) is required for these materials to be used in routine orthodontic practice.

Biomimetic adhesives: The bonding of the brackets to the tooth surface requires enamel preconditioning which leads to alteration in the enamel thickness and color. The durability and firmness of the bond between enamel surface and bracket base depends on adhesive material, preparation of tooth surface and brackets base retention. ${ }^{12}$ Zachrisson et al. reported that sandblasting also increases the bond strength to gold, porcelain and amalgam, 18 but all these manoeuvre would result in enamel damage and loss.

In the past, different types of materials were introduced which are closely related to the nature's pattern and has led to the creation of a separate division of biomimetic materials. Their essence can be appreciated by breaking the word into "bio", meaning life and "mimetic" which means imitating. ${ }^{12}$

Geckos, a kind of lizard, use the concept of "contact splitting" to balance their weight while they are upside down. This kind of adhesion goes well with dry surroundings. The example of mussel is fascinating for the researchers as it provides adhesion in wet environment. In this way, Geckel was introduced which is a mixture of adhesive components of Geckos and mussels and performs well in both dry and wet surroundings. ${ }^{12}$

The application of biomimetic adhesives in orthodontic science is by utilising the brackets with bases covered with L-3,4-dihydroxyphenylalanine (DOPA). DOPA is an important glue protein of mussels and provides sufficient bond strength with the enamel surface. ${ }^{19}$ Hence, enamel conditioning before bonding would not be required and less structural changes to enamel would be needed.

Self-cleaning materials: The plaque accumulation around brackets and tooth surfaces causes significant damage to the periodontium and subsequently leads to deleterious effects such as gingivitis, bone loss and white spot lesions. The introduction of such materials, which could easily flush away the organic and inorganic substances from the calcified surfaces and brackets would play a tremendous role in orthodontic material application. ${ }^{12}$

Over time, research has helped develop several biological micro and nano-structures which have beneficial properties. ${ }^{20}$ A good example is the lotus leaf, which grows in water. This is the only natural plant which is 
extremely hydrophobic in nature and stays clean in dirty water environment.20,21 Following the example of lotus leaf, self-cleaning materials have been introduced which show hydrophobic qualities. Introduction of titanium oxide nano-coating on the surface of aircrafts to increase the safety and durability also provides the selfcleaning effect. 12

In orthodontic materials, the photo-catalytic behaviour of titanium oxide with ultraviolet light is the centre of recognition in current time.22 The surface of nickel titanium archwire alloy has been changed to crystalline rutile by treating the alloy surface with titanium oxide film electrolytically and then with heat. ${ }^{12}$ Here a question arises, "Would this surface alteration of nickel titanium archwire result in significant change in the biomechanical properties of the alloy?" A question which is yet to be answered and a grey area which requires further research.

Biodegradable or bioresorbable miniimplants: The concept of biodegradable implants was introduced in the 1980's after extensive research for orthopedic purposes. ${ }^{23}$ The use of these materials was popularised due to the drawbacks of metallic fixation such as cosmetic deformity, palpability or wound dehiscence. ${ }^{24}$ Initially, fixation plates and screws were fabricated from polylactic and polyglycolic acid (PLA/PGA).25,26 These materials have the ability to break down into simpler compounds that can easily be excreted from the body. This makes them a valuable prospect for temporary anchorage devices used in orthodontic treatment. By altering the PLA/PGA ratio, we can change the degradation, excretion rate and biomechanical properties of these implants. ${ }^{24}$

The hesitation in widely adopting bioresorbable materials is the delayed resorption time. 27 Several authors $28-30$ have reported the time between 2-5 years for complete resorption of different materials. These polymer materials are overcoming the possibility of developing inflammatory response, infection and loosening of conventional temporary anchorage devices, but there is still a need to ensure safe elimination of the byproducts from the body. ${ }^{26}$ Perhaps, continued research will help in developing new materials that can be used and excreted from the body safely.

Fluoride releasing materials: The potential of orthodontic appliances to promote plaque accumulation and initiate the cariogenic process has led numerous innovations to tackle this complication. ${ }^{31}$ The application of fluoride varnishes before bonding and monthly application has shown reduced incidence of demineralisation. ${ }^{32,33}$

New materials, which are easy to apply such as titanium tetraflouride and sodium diamine fluoride, promise a reduction in caries. However, further studies are required to identify their anti-cariogenic properties. 34,35
Furthermore, a study using $\mathrm{CO}_{2}$ lasers and fluoride varnishes has also been conducted demonstrating the effect of laser therapy on the solubility of enamel. 36 Bonding materials such as compomers and resinmodified glass ionomer cements (RMGICs) have been shown to reduce the incidence of caries development but still require additional trials before they are widely used due to questionable bond strengths. 32,37

The conversion of hydroxyapatite to fluorapatite crystals contributes to the anti-cariogenicity imparted by fluoride. In addition, high quantities of fluoride in plaque is bactericidal and further prevents enamel breakdown. Casein Phosphopeptide-Amorphous Calcium Phosphate (CPPACP) maintains the level of phosphate and calcium in the plaque, therefore, it prevents the discharge of these elements and further encourages remineralisation. ${ }^{32,38}$

Methods to maintain high concentrations of fluoride in the oral cavity in orthodontic patients has also included slow releasing devices, chewing gums and elastomers. Each of these has shown increased amounts of fluoride in the oral cavity. However, incorporation of fluoride has resulted in significant alteration in the mechanical properties of these materials. ${ }^{32,39-41}$ Multiple trials need to be undertaken to establish standard guidelines for the usage of fluoride for each orthodontic patient so that deleterious and iatrogenic effects such as development of white spot lesions can be minimised.

Recommendations: Smart biocompatible materials, adhesives, brackets and archwires will soon become the part of the routine orthodontic armamentarium due to continuing in vitro trials. New advances and technologies of biomedical engineering and predominantly the use of nanotechnology for material construction and manufacture are being used for headway in material sciences. The application of tissue engineering principles for dental, skeletal and soft tissues, and development of nanostructured and biomimetic materials should be prominent areas of forthcoming research. Orthodontic faculties in research-oriented institutions need to approach faculty in biomedical engineering or bioengineering departments and point out the opportunities that are available for collaboration and advances in both areas.

\section{CONCLUSION}

This review has discussed the biomedical materials, their current trends, orthodontic implications and future orthodontic perspectives. For customisation of orthodontic appliances, the interaction between orthodontists and biomedical engineers is of profound importance to serve the patients in a better way with least undesirable complications of the armamentarium.

\section{REFERENCES}

1. Nikolai RJ. Bioengineering analysis of orthodontic mechanics. In: Lea \& Febiger, Philadelphia; 1985:55. 
2. Kusy RP, Greenberg AR. Effects of composition and cross section on the elastic properties of orthodontic wires. Angle Orthod 1981; 51:325-41.

3. Eliades T, Eliades G, Silikas N, Watts DC. Tensile properties of orthodontic elastomeric chains. Euro J Orthod 2004; 26:157-62.

4. Wong BH. Invisalign A to Z. Am J Orthod Dentofacial Orthop 2002; 121:540-1.

5. Fuck LM, Wiechmann D, Drescher D. Comparison of the initial orthodontic force systems produced by a new lingual bracket system and a straight-wire appliance. J Orofac Orthop 2005; 66:363-76.

6. Kocadereli I, Canay S, Akça K. Tensile bond strength of ceramic orthodontic brackets bonded to porcelain surfaces. Am J Orthod Dentofacial Orthop 2001; 119:617-20.

7. Behl M, Lendlein A. Shape-memory polymers. Mater Today 2007; 10:20-8.

8. Jung YC, Cho JW. Application of shape memory polyurethane in orthodontics. J Mater Sci Mater Med 2010; 21:2881-6.

9. Ward Small IV, Singhal P, Wilson TS, Maitland DJ. Biomedical applications of thermally activated shape memory polymers. J Mater Chem 2010; 20:3356-66.

10. Nakasima A, Hu JR, Ichinose M, Shimada H. Potential application of shape memory plastic as elastic material in clinical orthodontics. Euro J Orthod 1991; 13:179-86.

11. Gorna K, Gogolewski S. The effect of gamma radiation on molecular stability and mechanical properties of biodegradable polyurethanes for medical applications. Polym Degrad Stab 2003; 79:465-74.

12. Eliades T. Orthodontic material applications over the past century: Evolution of research methods to address clinical queries. Am J Orthod Dentofacial Orthop 2015; 147:224-31.

13. Gündüz E, Zachrisson BU, Hönigl KD, Crismani AG, Bantleon HP. An improved transpalatal bar design. Part I. Comparison of moments and forces delivered by two bar designs for symmetrical molar derotation. Angle Orthod 2003; 73:239-43.

14. Badawi HM, Toogood RW, Carey JP, Heo G, Major PW. Threedimensional orthodontic force measurements. Am J Orthod Dentofacial Orthop 2009; 136:518-28.

15. Lapatki BG, Bartholomeyczik J, Ruther P, Jonas IE, Paul O. Smart bracket for multi-dimensional force and moment measurement. J Dent Res 2007; 86:73-8.

16. Chen J, Dong Q, Ma X, Fan T, Lei Y. Repetitive biomimetic selfhealing of $\mathrm{Ca} 2+$ induced nanocomposite protein hydrogels. Sci Rep 2016; 6:30804.

17. Phadke A, Zhang C, Arman B, Hsu CC, Mashelkar RA, Lele AK, et al. Rapid self-healing hydrogels. Proc Natl Acad Sci 2012; 109:4383-8.

18. Zachrisson BU, Buyukyilmaz T, Zachrisson YO. Improving orthodontic bonding to silver amalgam. Angle Orthod 1995; 65:35-42.

19. Hamming LM, Fan XW, Messersmith PB, Brinson LC. Mimicking mussel adhesion to improve interfacial properties in composites. Compos Sci Technol 2008; 68:2042-8.

20. Lalthe S, Terashina C, Nakata K, Fujishima A. Super-hydrophobic surfaces developed by mimicking hierarchical surfaces morphology of lotus leaf. Molecules 2014; 19:4256-83.

21. Xu Q, Zhang W, Dong C, Sreeprasad TS, Xia Z. Biomimetic self-cleaning surfaces: Synthesis, mechanism and application. J Royal Soc Interface 2016; 13:20160300.

22. Horiuchi Y, Horiuchi M, Hanawa T, Soma K. Effect of surface modification on the photocatalysis of Ti-Ni alloy in orthodontics. Dent Mater J 2007; 26:924-9.

23. Rokkanen P, Bostman O, Makela EA, Hirvensalo E, Partio EK, Vihtonen $\mathrm{K}$, et al. Absorbable devices in the fixation of fractures. J Trauma Acute Care Surg 1996; 40:123-27.

24. Glatzmaier J, Wehrbein H, Peter D. Biodegradable implants for orthodontic anchorage. A preliminary biomechanical study. Euro J Orthod 1996; 18:465-9.

25. Gunatillake Pa, Adhikari R, Gadegaard N. Biodegradable synthetic polymers for tissue engineering. Eur Cells Mater 2003; 5:1-16.

26. Ulery BD, Nair LS, Laurencin CT. Biomedical applications of biodegradable polymers. J PolymSci B Polym Phys 2011; 49:832-64.

27. Little $\mathrm{H}$, Clarke SA, Cunnigham E, Buchanan F. Processinduced degradation of bioresorbable PDLGA in bone scaffold production. J Mater Sci Mater Med 2018; 29:14.

28. Hutmacher DW, Schantz T, Zein I, Ng KW, Teoh SH, Tan KC. Mechanical properties and cell cultural response of polycaprolactone scaffolds designed and fabricated via fused deposition modeling. J Biomed Mater Res 2001; 55:203-16.

29. Zein I, Hutmacher DW, Tan KC, Teoh SH. Fused deposition modeling of novel scaffold architectures for tissue engineering applications. Biomaterials 2002; 23:1169-85.

30. Choong C, Triffitt JT, Cui ZF. Polycaprolactone scaffolds for bone tissue engineering effects of a calcium phosphate coating layer on. Food Bioprod Process 2004; 82:117-25.

31. Gong Y, Lu J, Ding Xiajun. Clincal, microbiological, and immunological factors of orthodontic treatment-induced gingival enlargement. Am J Orthod Dentofacial Orthop 2011; 140:58-64.

32. Graber LW, Vanarsdall RL, Vig KW, Huang GJ. Orthodontics: Current Principles and Techniques. Elsevier Health Sciences; 2016.

33. Justus R. Prevention of white spot lesions during orthodontic treatment. Clin Dent Rev 2018; 2:1.

34. Bridi EC, do Amaral FLB, Franca FMG, Tursi CP, Basting RT. Influence of dentin pretreatment with $2.5 \%$ titanium tetraflouride on inhibiting caries at the tooth-restoration interface in situ. Arch Oral Biol 2018; 86:51-7.

35. Burgess JO, Vaghela PM. Silver diamine fluoride: A successful anticarious solution with limits. Adv Dent Res 2018; 29:131-4.

36. Chuan $X$, Chen $L$. Effects of enamel surface preparation on bonding strength of resin-modified glass ionomer cement: An in vitro study. J Adhes Sci Technol 2018; 31:2300-11.

37. Mahmoudzadeh M, Soufi LR, Farhadian N, Jamalian SF, Akbaradeh M, Momeni M, et al. Effect of $\mathrm{CO}_{2}$ laser and fluoride varnish application of microhardness of enamel surface around orthodontic brackets. J Lasers Med Sci 2017; 9:43-9.

38. Nhu NV, Hong TP, Le AQ, Minh ST, Thu PN. The effect of casein phosphopeptide-amorphous calcium fluoride phosphate on the remineralization of artificial caries lesions: an in vitro study. J Dent Indonesia 2017; 24:45-9.

39. Banks PA, Chadwick SM, Asher-McDade C, Wright JL. Fluoride-releasing elastomerics: A prospective controlled clinical trial. Eur J Orthod 2000; 22:401-7.

40. Mattick CR, Mitchell L, Chadwick SM, Wright J. Fluoridereleasing elastomeric modules reduce decalcification: A randomized controlled trial. $J$ Orthod 2001; 28:217-9.

41. Pessan JP, Al-Ibrahim NS, Buzalaf MA, Toumba KJ. Slowrelease fluoride devices: A literature review. J Appl Oral Sci $2008 ; 16: 238-46$ 